\title{
High concordance of TMPRSS-ERG fusion between primary prostate cancer and its lymph node metastases
}

\author{
FRANZISKA BRANDI $^{1}$, KATHARINA GRUPP $^{2}$, CLAUDIA HUBE-MAGG $^{1}$, MARTINA KLUTH $^{1}$, \\ DAGMAR LANG $^{1}$, SARAH MINNER ${ }^{1}$, CHRISTINA MÖLLER-KOOP $^{1}$, MARKUS GRAEFEN $^{3}$, \\ HANS HEINZER $^{3}$, MARIA CHRISTINA TSOURLAKIS ${ }^{1}$, CORINNA WITTMER $^{1}$, FRANK JACOBSEN ${ }^{1}$, \\ HARTWIG HULAND $^{3}$, STEFAN STEURER ${ }^{1}$, PATRICK LEBOK ${ }^{1}$, ANDREA HINSCH ${ }^{1}$, \\ WALDEMAR WILCZAK ${ }^{1}$, THORSTEN SCHLOMM ${ }^{3,4}$ and RONALD SIMON ${ }^{1}$ \\ ${ }^{1}$ Institute of Pathology; ${ }^{2}$ General, Visceral and Thoracic Surgery Department and Clinic; \\ ${ }^{3}$ Martini-Clinic, Prostate Cancer Center; ${ }^{4}$ Department of Urology, Section for Translational Prostate Cancer Research, \\ University Medical Center Hamburg-Eppendorf, Hamburg D-20246, Germany
}

Received September 21, 2017; Accepted August 2, 2018

DOI: $10.3892 / 01.2018 .9417$

\begin{abstract}
Approximately $50 \%$ of prostate cancer types harbor the transmembrane protease, serine 2: Erythroblast transformation-specific-related gene (ERG) fusion, resulting in oncogenic expression of the ERG transcription factor. ERG represents an attractive target for potential future anticancer therapy in advanced and metastatic prostate cancer. To better understand whether the analysis of the primary cancer is sufficient to estimate the ERG expression status of the lymph node metastases, the present study examined patterns of immunohistochemical ERG expression in a tissue microarray created from multiple primary and metastatic sites of 77 prostate cancer tissues. Among the identified tumor types, $80 \%$ were either entirely ERG-positive (38\%) or ERG-negative (42\%) across all (at least 9) analyzed different tumor sites. The results were heterogeneous in $20 \%$ of the tumor types and typically resulted from small ERG-negative areas within otherwise ERG-positive tumor types. Comparison of the ERG expression status in 51 primary cancer types with at least three interpretable lymph node metastases revealed an entirely identical ERG status in all tumor sites in $75 \%$ of the cases, including 16 ERG-positive and 22 ERG-negative cancer types.
\end{abstract}

Correspondence to: Dr Ronald Simon, Institute of Pathology, University Medical Center Hamburg-Eppendorf, Martinistrasse 52, Hamburg D-20246, Germany

E-mail: r.simon@uke.de

Abbreviations: ERG, erythroblast transformation-specific-related gene; ICH, immunohistochemistry; TMPRSS2:ERG, transmembrane protease, serine 2: erythroblast transformation-specific-related gene fusion; TMA, tissue microarray

Key words: prostate cancer, transmembrane protease, serine 2: erythroblast transformation-specific-related gene fusion, nodal metastasis, immunohistochemistry, tissue microarray
The remaining 13 cancer types exhibited ERG heterogeneity within the primary tumor, while all metastases had an identical (12 positive and 1 negative) ERG status. The results of the present study revealed a high degree of concordance of the ERG expression status between primary prostate cancer types and their lymph node metastases. Therefore, potential anti-ERG therapy may also be effective against lymph node metastases in the majority of cases of ERG-positive metastatic prostate cancer.

\section{Introduction}

Prostate cancer is the most frequent malignancy in men (1). Approximately $10-20 \%$ of patients suffer from cancer invading into the lymph system at the time of diagnosis (2-4). Finding tumor cells in a lymph node is unequivocal proof of dissemination and is associated with poor patient prognosis (5-9). Thus, novel therapies are needed to effectively target metastatic cancer cells.

Approximately $50 \%$ of prostate cancers harbor a gene fusion linking the androgen-regulated gene transmembrane protease, serine 2 (TMPRSS2) with transcription factors of the erythroblastosis virus E26 transforming sequence (ETS) family, typically erythroblast transformation-specific-related gene (ERG) (10). The TMPRSS2:ERG fusion protein may be an optimal target for a novel therapy, as it is highly specific for prostate cancer cells. In addition, potential anti-TMPRSS2:ERG therapy is unlikely to have major side effects, since this fusion protein is absent in normal tissues. Recent advances in the delivery of inhibitory RNAs or peptides to human cancer raise the possibility that anti-TMPRSS2:ERG therapy may become available in the future (11-14).

It has been recently demonstrated that the TMPRSS2:ERG fusion typically occurs early during tumor development and is often homogeneously distributed across the cancer bulk (15-18). However, we also observed that up to $60 \%$ of ERG-positive cancers may at the least have small areas lacking ERG expression $(18,19)$. This raises the question whether lymph 
node metastasis arises from ERG-negative or -positive areas, and whether the ERG status of the primary cancer represents the ERG status of the lymph node metastasis. In a recent study, discrepant ERG findings were observed in the lymph nodes of $30 \%$ of 84 prostate cancers (20). Such differences in the ERG status between primary and metastatic tumor sites would challenge the concept of anti-ERG therapy. We herein performed a thorough ERG-mapping study in 77 prostate cancers exhibiting lymph node involvement at the time of diagnosis. We constructed a tissue microarray (TMA) with 20 spots per primary cancer and all tumor-containing lymph nodes for maximal representation of the tumor bulk. Our findings demonstrated a high degree of concordance of the ERG expression status between primary prostate cancers and their lymph node metastases, and little intratumoral heterogeneity.

\section{Materials and methods}

Tissue samples. The prostate cancer heterogeneity TMA consisted of 1,727 prostate cancer tissue spots and 80 control spots from normal tissue (lung, liver, skin, lymph node and kidney) distributed across 4 paraffin blocks, all derived from 77 patients who underwent radical prostatectomy at the UKE between 2009 and 2010, who were found to have lymph node metastases. A total of 24-61 primary tumor-containing blocks and 1-16 blocks from the corresponding lymph node metastases were collected from each patient. For the TMA construction, 20 0.6-mm punches were collected from each primary tumor, with one additional punch from each of the corresponding metastatic lymph nodes. If possible, all 20 primary cancer tissue punches were collected from different paraffin blocks. The number of sampled lymph nodes per patient was as follows: 1 node in 4 patients, 2 nodes in 17 patients, 3 nodes in 30 patients, 4 nodes in 7 patients, 5 nodes in 5 patients, 6 nodes in 4 patients, 7 nodes in 5 patients, 10 nodes in 1 patient, 11 nodes in 1 patient, 14 nodes in 2 patients, and 16 nodes in 1 patient. All cancers were unifocal tumors according to the criteria of Wise et al (21): The tumor areas were defined as part of a single focus if they were within $3 \mathrm{~mm}$ of each other in any section or within $4 \mathrm{~mm}$ on adjacent sections.

Immunohistochemistry (IHC). IHC analysis of ERG was performed as previously described (17). Rabbit recombinant monoclonal ERG antibody (clone EPR3864, dilution 1:450; Epitomics) was used and visualized with DAKO EnVision (Dako Diagnostics AG, Zug, Switzerlan). Freshly cut TMA sections were analyzed in one day and in one experiment. The sections were deparaffinized and exposed to heat-induced antigen retrieval for $5 \mathrm{~min}$ at $121^{\circ} \mathrm{C}$ in citrate buffer $(\mathrm{pH} 7.8)$. Only nuclear ERG staining was scored. Staining intensity was assessed on a scale of 0 (negative), 1 (weak), 2 (moderate), and 3 (strong) (Fig. 1). Any detectable staining $(\geq 1)$ was considered as ERG-positive.

\section{Results}

Of the 77 different cancers, 69 had at least 9 interpretable ERG results in the 20 tissue spots obtained from the primary cancer: 12 cancers had 20 interpretable spots, 21 cancers had 19 interpretable spots, 35 cancers had 10-18 interpretable spots, and 1 cancer had 9 interpretable spots. At least one lymph node metastasis was analyzable from 70 cancers, whereas 54 cancers had at least three analyzable lymph node metastases.

ERG heterogeneity in the primary cancer. Of the 69 primary cancers with at least 9 interpretable tissue spots, $14(20 \%)$ were heterogeneous (Fig. 2). Heterogeneity was found in every Gleason score. Representative images of heterogeneous cases with single discrepant ERG-positive or ERG-negative spots are shown in Fig. 3. An identical ERG result was found in all tissue spots of 55/69 (80\%) tumors, of which 26/69 (38\%) primary cancers were homogeneously ERG-positive and 29/69 (42\%) homogeneously ERG-negative.

Comparison between primary cancers and their lymph node metastasis. This analysis was restricted to the subset of 49 cancers with at least 9 interpretable tumor spots in the primary cancer and with at least 3 interpretable lymph node metastases (Fig. 4). In the subset of primary cancers with a homogeneous ERG staining result, 38/38 (100\%) were identical with their metastasis. In the subset of primary cancers with a heterogeneous ERG staining result, $12 / 13(92 \%)$ cancers were ERG-positive and 1/13 (8\%) was ERG-negative.

\section{Discussion}

The results of the present study suggest a high degree of concordance $(50 / 51,98 \%)$ of ERG expression between primary cancer and lymph node metastasis. We found 40/69 (58\%) of the cancers to be ERG-positive, in accordance with earlier reports of 40-60\% ERG positivity in studies with 13-317 prostate cancers, which were analyzed as conventional large sections $(15,17,18,22,23)$ or as TMA spots $(19,24,25)$. For example, we found $45 \%$ ERG-positive cases in a recent study of all tumor-containing conventional large sections of 317 prostate cancers obtained from 125 patients (18), and $55 \%$ ERG-positive cancers in a TMA study with one $0.6-\mathrm{mm}$ tissue spot per cancer using the same ERG antibody and IHC protocol (19). Thus, the fraction of ERG-positive cancer is independent from the amount of tissue analyzed.

Whether ERG activation is associated with prostate cancer aggressiveness is a matter of debate. For example, certain studies have suggested an association between ERG expression and advanced tumor stage $(26,27)$, high Gleason grade (26), or poor patient prognosis $(26,28)$, while other studies, including ours, could not confirm such findings $(19,29,30)$. In our earlier TMA study on 2,800 cancers, ERG expression was identified in 55\% non-metastatic and 54\% metastatic cancers (19). The similar rate of ERG positivity (58\%) in the present high-risk cancer series selected for lymph node metastasis provides strong additional evidence against a relevant role of ERG in tumor aggressiveness.

While $80 \%$ of the primary cancers were homogeneous in terms of ERG expression, this percentage increased to $98 \%$ for the metastases. These data suggest that the ERG expression status is typically preserved during metastatic spread. Similar findings were reported by an earlier heterogeneity study on 86 primary prostate cancers with matched lymph node metastases (20), where $71 \%$ of the cancers had identical ERG findings in at least 2 samples, each obtained from the primary and 
A
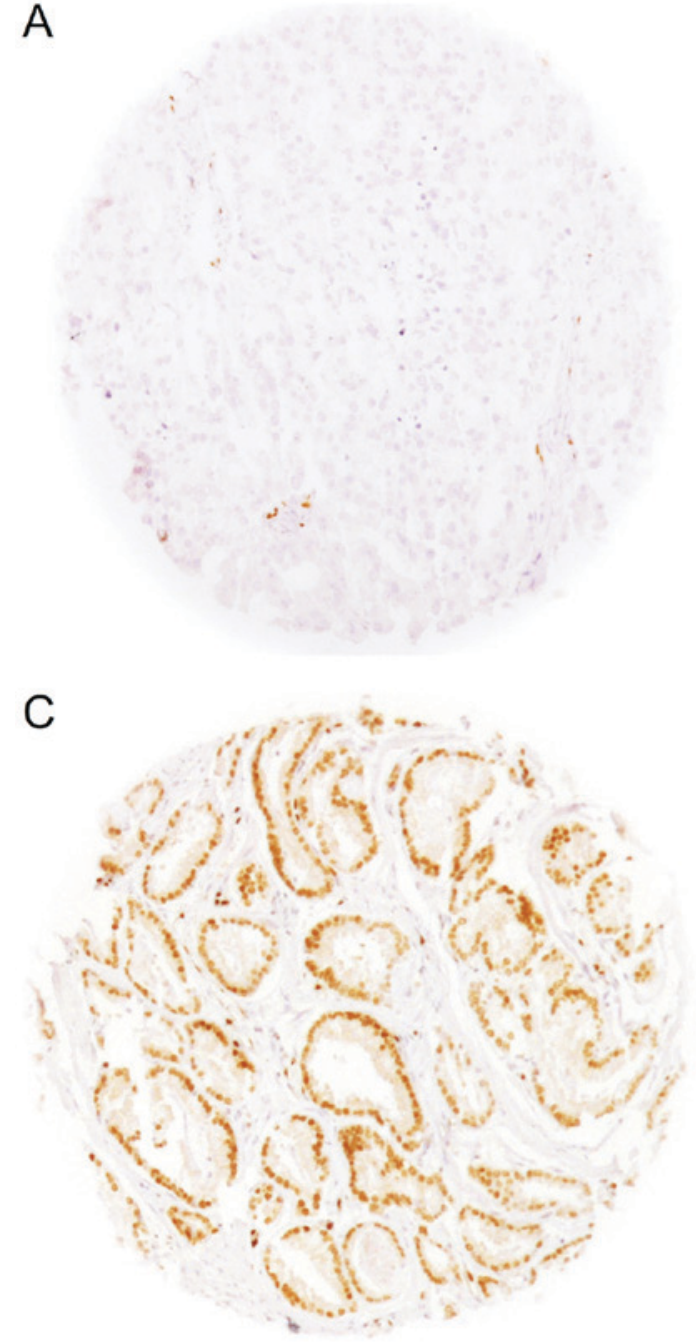

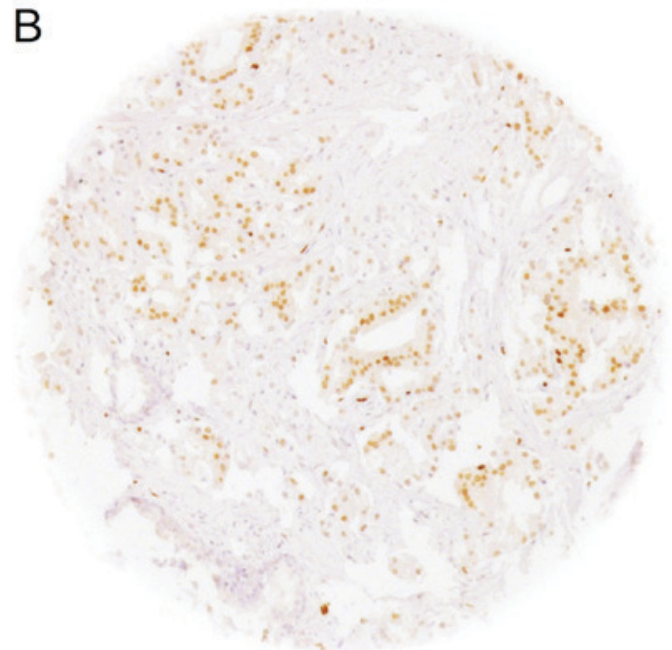

B

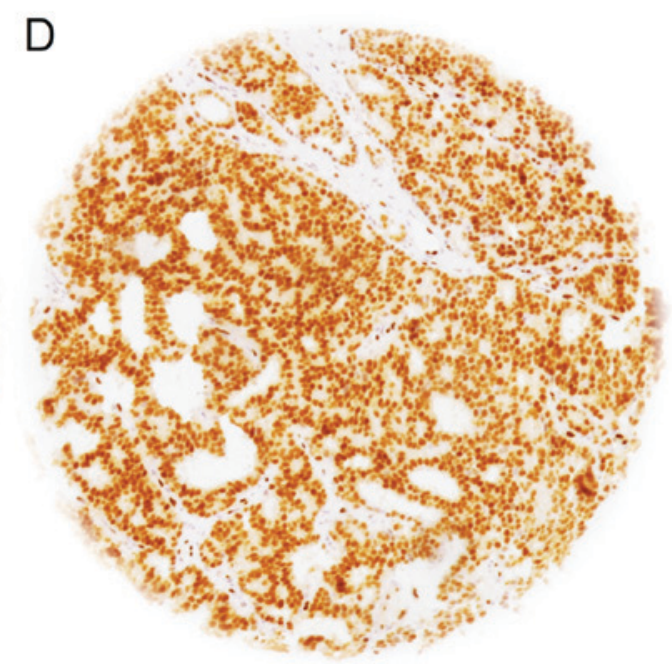

Figure 1. Representative images of ERG staining intensities in homogeneous cases: (A) Negative, (B) weak, (C) moderate and (D) strong. Original spot size $(0.6 \mathrm{~mm}) \times 100$ magnification. ERG, protein encoded by the erythroblast transformation-specific-related gene.

metastatic tumor sites. Smaller studies on 13 (31) and 26 (32) cancers reported a concordance rate between 77 and $100 \%$.

In our study, discrepant findings between the primary and metastatic sites were yielded from cases with heterogeneous ERG expression in the primary cancer. However, it should be noted that 8 of 14 primary cancers with heterogeneous ERG staining were rated as discrepant only because one individual TMA spot yielded a different ERG result. It remains uncertain whether such cases represent true heterogeneity. Although we strictly followed the guidelines to identify individual foci, it cannot be excluded that some discrepant findings are due to collision of cancer foci that cannot be distinguished histologically, or that local variations of staining account for rare false-negative findings.

Although some heterogeneous findings may be associated with technical issues, this is not always the case. In order to ensure a high representativeness of the analyzed cancers, we limited our study to tumors with at least 9 analyzable primary tissue spots and at least 3 analyzable metastases. Identifying $\geq 2$ discrepant spots in $\sim 7 \%$ of primary cancers suggests that a fraction of cancers may harbor true intrafocal heterogeneity. Such findings support our hypothesis that ERG fusion may also develop at a later time after the cancer has been established. We have already made this observation in our earlier study on conventional large sections, where we found $42 \%$ of ERG-positive tumors to have at least small ERG-negative areas (17). The higher heterogeneity rate in the latter study is most likely attributed to the higher amount of cancer tissue that can be analyzed in conventional large sections. Our findings raise the possibility that a relevant fraction of ERG-positive prostate tumors may initially develop as ERG-negative. This is of interest in the light of earlier discussions on whether ERG fusion is sufficient to initiate prostate cancer $(33,34)$. Based on our data, it may be hypothesized that ERG activation represents a very early progression event rather than a cancer-initiating alteration in several cases. This view is further supported by studies reporting that mouse prostate with forced ERG expression displayed only subtle morphological changes (34), and that additional alterations, such as loss of the PTEN tumor suppressor, was required to develop invasive cancer (34).

The limitation of the present study is that it is purely descriptive and does not involve any comparisons between molecular groups that would allow for statistical testing. The same is the case for the comparison of the ERG status in primary cancers and their metastases: All cancers are metastatic so that there is no comparison between subsets that are defined by specific molecular or histologic features. 
\begin{tabular}{ll|l|l|l|l|l|l|l|l|l|l|l|l|l|} 
Case Nr. 71 & 8 & 44 & 67 & 41 & 22 & 32 & 39 & 76 & 15 & 30 & 64 & 26 & 10 \\
\hline
\end{tabular}

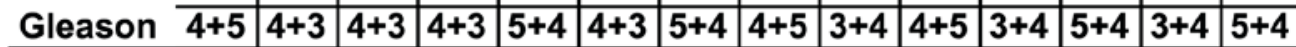

\begin{tabular}{|c|c|c|c|c|c|c|c|c|c|c|c|c|c|c|}
\hline 0 & s & S & s & os & pos & os & pos & pos & n.a. & eg & n.a. & neg & neg & \\
\hline & & $s$ & & & $S$ & $S$ & S & $\mathrm{s}$ & s & S & a. & eg & eg & \\
\hline & & s & os & & n.a. & $s$ & pos & Ds & $\mathrm{gg}$ & DS & pos & eg & os & \\
\hline & & is & os & S & os & s & os & DS & os & eg & a. & os & $\mathrm{g}$ & \\
\hline & S & os & os & S & pos & os & a. & os & os & a. & pos & neg & eg & \\
\hline & s & os & s & ss & pos & os & pos & pos & os & SS & a. & os & $\mathrm{g}$ & \\
\hline & $S$ & S & os & s & $\mathbf{S}$ & os & pos & pos & neg & r & pos & eg & s & \\
\hline & $S$ & S & s & pos & pos & pos & pos & n.a. & neg & a. & neg & pos & os & \\
\hline & $S$ & pos & os & os & pos & pos & pos & pos & pos & pos & n.a. & eg & a. & \\
\hline & S & pos & pos & pos & neg & pos & pos & neg & pos & a. & n.a. & leg & eg & \\
\hline & os & pos & neg & a. & pos & pos & neg & pos & pos & neg & pos & pos & neg & \\
\hline & os & pos & os & pos & pos & pos & pos & pos & pos & os & neg & pos & g & \\
\hline & os & neg & os & pos & pos & a. & pos & pos & pos & pos & pos & pos & g & \\
\hline & os & pos & os & pos & pos & n.a. & pos & pos & pos & pos & pos & pos & eg & \\
\hline & SS & $n$ & n.a. & pos & pos & pos & a. & pos & pos & a. & pos & pos & neg & \\
\hline & S & pos & os & pos & $p$ & pos & os & pos & pos & a. & pos & pos & ieg & \\
\hline & SS & pos & os & pos & pos & n.a. & pos & pos & pos & pos & neg & pos & n.a. & \\
\hline Spot 18 & s & pos & os & pos & pos & pos & n.a. & pos & pos & pos & pos & pos & n.a. & \\
\hline Spot 19 & os & pos & os & pos & pos & pos & pos & neg & pos & pos & neg & pos & n.a. & \\
\hline & Ss & os & ss & neg & $n$ & neg & n.a. & pos & pos & po: & pos & pos & n.a. & \\
\hline
\end{tabular}

\begin{tabular}{|c|c|c|c|c|c|c|c|c|c|c|c|c|c|c|}
\hline N 1 & s & pos & pos & pos & pos & pos & n.a. & pos & pos & pos & pos & pos & pos & 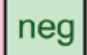 \\
\hline$\sqrt{ } 2$ & & pos & pos & pos & pos & pos & n.a. & pos & pos & pos & n.a. & pos & pos & \\
\hline$\sqrt{ } 3$ & & os & pos & os & pos & pos & pos & pos & pos & pos & pos & pos & pos & \\
\hline$\sqrt{ } 4$ & os & pos & n.a. & pos & pos & pos & n.a. & pos & pos & n.a. & pos & pos & ก.а. & \\
\hline V 5 & os & pos & n.a. & pos & pos & n.a. & n.a. & n.a. & pos & pos & n.a. & n.a. & n.a. & \\
\hline V 6 & ss & pos & n.a. & a. & pos & pos & n.a. & n.a. & n.a. & pos & n.a. & n.a. & n.a. & \\
\hline ง 7 & os & n.a. & n.a. & a. & pos & n.a. & .a. & n.a. & n.a. & n.a. & a. & n.a. & n.a. & \\
\hline V 8 & os & n.a. & n.a & n.a. & os & n.a. & n.a. & a. & n.a. & a. & a. & n.a. & n.a. & \\
\hline 19 & n.a. & n.a. & n.a. & n.a. & pos & n.a. & a. & a. & n.a. & n.a. & a. & n.a. & n.a. & \\
\hline 1 & a. & n.a. & n.a. & n.a. & pos & n.a. & n.a. & n.a. & n.a. & n.a. & n.a. & n.a. & n.a. & \\
\hline 11 & .a. & n.a. & n.a. & n.a. & pos & n.a. & n.a. & n.a. & n.a. & n.a. & n.a. & n.a. & n.a. & \\
\hline $\mathrm{N} 12$ & n.a. & n.a. & n.a. & n.a. & pos & n.a. & n.a. & n.a. & n.a. & n.a. & n.a. & n.a. & n.a. & \\
\hline
\end{tabular}

Figure 2. Fourteen primary prostate cancer cases and their Gleason grade with heterogeneous ERG findings. Red color indicates ERG-positive primary cancer spots, and green color ERG-negative primary spots. Grey color indicates non-analyzable spots. ERG, protein encoded by the erythroblast transformation-specific-related gene. 

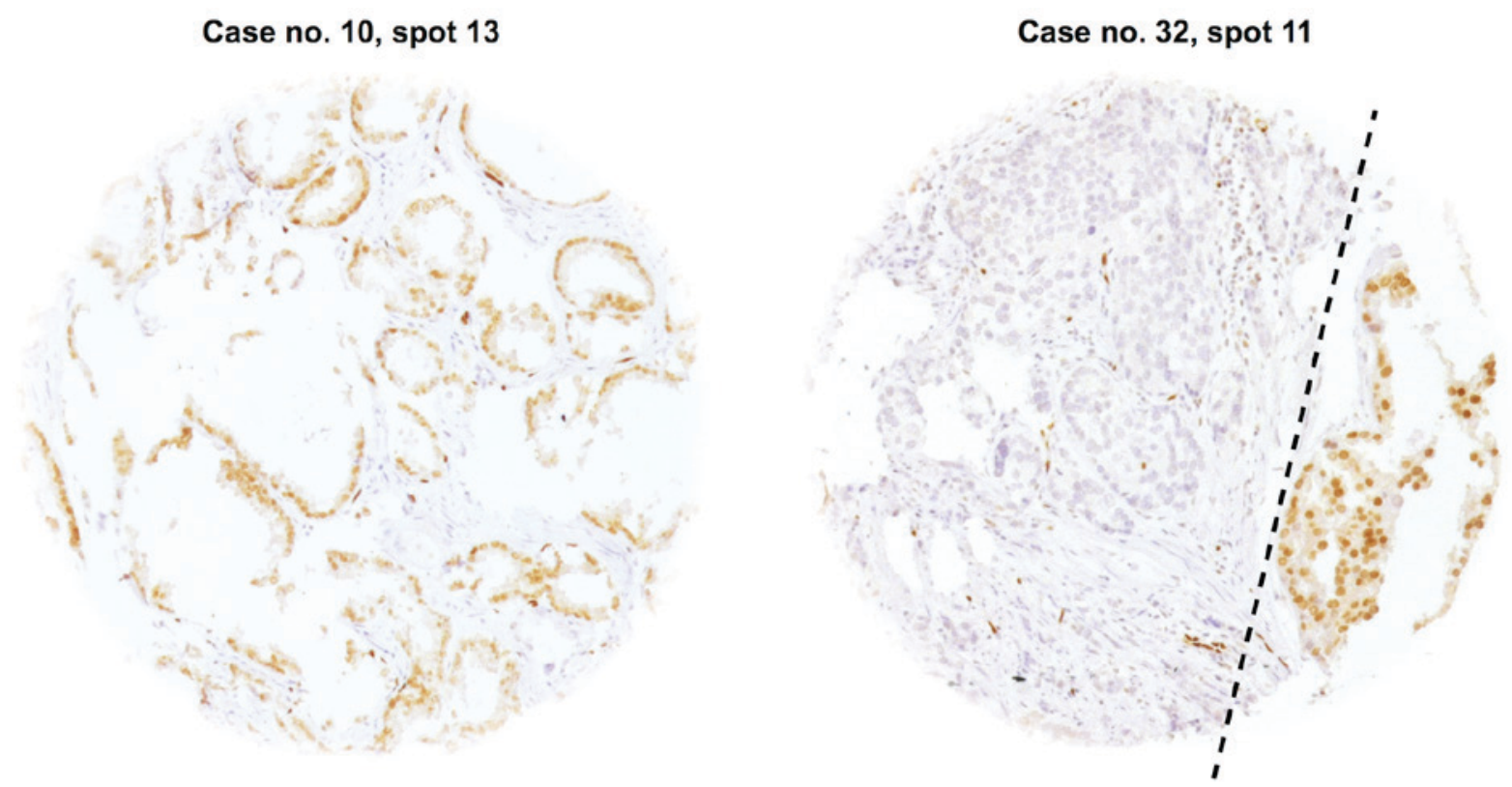

Figure 3. Representative pictures of cases from Fig. 2 with heterogeneous tumor samples e.g., case no. 10 the single positive spot 13 and case no. 32 the single negative spot 11. Note the ERG-positive tumor cells to the right from the dotted line in this spot. Original spot size $(0.6 \mathrm{~mm})$ x $100 \mathrm{magnification}$.

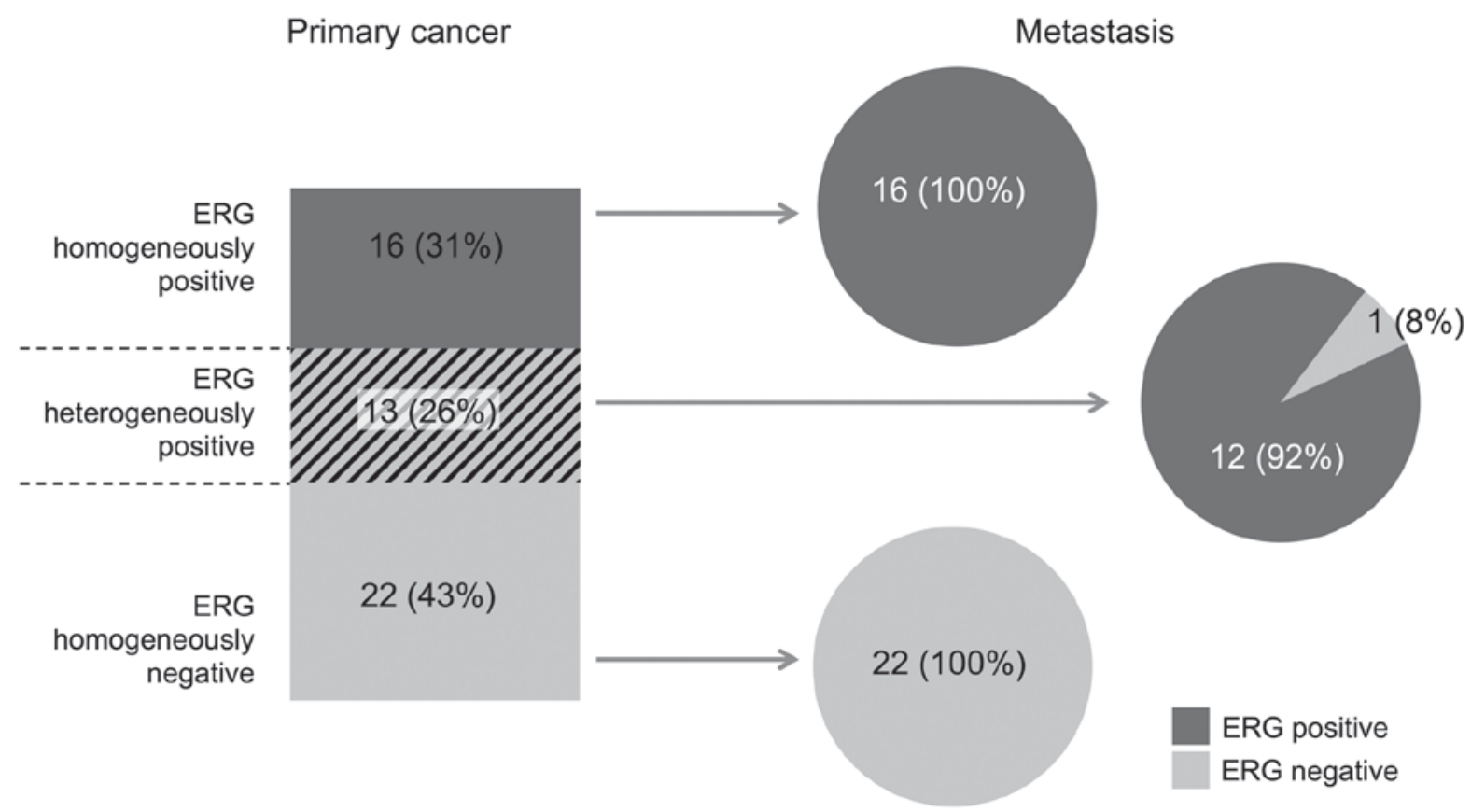

Figure 4. Comparison of the ERG status in 49 primary cancers with matched lymph node metastases. ERG, protein encoded by the erythroblast transformation-specific related gene.

The TMPRSS2:ERG fusion oncogene is unique to $50 \%$ of prostate cancers, making it an attractive target for highly specific anticancer therapy. To date, no TMPRSS2:ERG-specific drug has been developed, but recent advances suggest that gene silencing with small interfering RNAs (siRNAs) or peptides may become an option in the future $(11,13,14)$. For example, in a xenograft tumor mouse model, knockdown of ERG overexpression strongly inhibited tumor growth already in the first week of treatment, and partially restored tumor cell differentiation without any signs of toxicity (11). The typically homogeneous ERG expression across all primary and metastatic tumor cells in ERG-positive prostate cancer suggests that anti-TMPRSS2:ERG therapy may prove to be highly effective in the future. In addition, the homogenous staining makes it highly likely that ERG-positive cancers will be reliably identified through analysis of prostate biopsies.

In summary, the results of our study demonstrated a high degree of concordance of ERG expression between primary prostate cancers and their lymph node metastases, with some intratumoral heterogeneity. Analysis of small samples of the cancer, such as needle biopsies, may be sufficient to select 
patients for putative anti-TMPRSS2:ERG therapy, should it become available in the future.

\section{Acknowledgements}

The authors would like to thank Mrs. Janett Lütgens, Mrs. Sünje Seekamp and Mrs. Inge Brandt for their technical assistance.

\section{Funding}

No funding was received.

\section{Availability of data and materials}

All data generated or analyzed during this study are included in this published article.

\section{Authors' contributions}

FB, KG, RS and TS designed the study and drafted the manuscript. HHe, HHu and MG participated in study design. SM, MCT, CW, FJ, CF and SS performed immunohistochemical analysis and scoring. MK and RS participated in pathology data analysis. CHM and RS performed statistical analysis. DL, CMK, AH and PL participated in data interpretation and helped to draft the manuscript. WW participated in data interpretation. All authors read and approved the final manuscript.

\section{Ethics approval and consent to participate}

The Ethics Committee of the Ärztekammer Hamburg approved the study protocol (approval no. WF-049/09). According to local laws (HmbKHG §12a), patient informed consent was not required. Patient records/information were anonymized and de-identified prior to analysis. All procedures have been performed in compliance with the principles outlined in the Helsinki Declaration.

\section{Patient consent for publication}

Not applicable.

\section{Competing interests}

The authors declare that they have no competing interests.

\section{References}

1. Jemal A, Bray F, Center MM, Ferlay J, Ward E and Forman D: Global cancer statistics. CA Cancer J Clin 61: 69-90, 2011.

2. Cai T, Nesi G, Tinacci G, Giubilei G, Gavazzi A, Mondaini N, Zini $\mathrm{E}$ and Bartoletti R: Clinical importance of lymph node density in predicting outcome of prostate cancer patients. J Surg Res 167: 267-272, 2011.

3. Bader P, Burkhard FC, Markwalder R and Studer UE: Disease progression and survival of patients with positive lymph nodes after radical prostatectomy. Is there a chance of cure? J Urol 169: 849-854, 2003

4. Weckermann D, Dorn R, Trefz M, Wagner T, Wawroschek F and Harzmann R: Sentinel lymph node dissection for prostate cancer: Experience with more than 1,000 patients. J Urol 177: 916-920, 2007.
5. Gervasi LA, Mata J, Easley JD, Wilbanks JH, Seale-Hawkins C, Carlton CE Jr and Scardino PT: Prognostic significance of lymph nodal metastases in prostate cancer. J Urol 142: 332-336, 1989.

6. Cheng L, Zincke H, Blute ML, Bergstralh EJ, Scherer B and Bostwick DG: Risk of prostate carcinoma death in patients with lymph node metastasis. Cancer 91: 66-73, 2001.

7. Zwergel U, Lehmann J, Wullich B, Schreier U, Remberger K, Zwergel $\mathrm{T}$ and Stoeckle M: Lymph node positive prostate cancer: Long-term survival data after radical prostatectomy. J Urol 171: 1128-1131, 2004

8. Fleischmann A, Rocha C, Schobinger S, Seiler R, Wiese B and Thalmann GN: Androgen receptors are differentially expressed in Gleason patterns of prostate cancer and down-regulated in matched lymph node metastases. Prostate 71: 453-460, 2011.

9. Ko K, Jeong IG, Choi WS, Lim JH, Suh JH, Ku JH, Park Y, Moon KC, Kim HH, Kim CS and Kwak C: Effect of Gleason scores of lymph node metastases on prognosis of patients with prostate cancer. Int J Clin Exp Pathol 7: 6141-6148, 2014.

10. Tomlins SA, Rhodes DR, Perner S, Dhanasekaran SM, Mehra R, Sun XW, Varambally S, Cao X, Tchinda J, Kuefer R, et al: Recurrent fusion of TMPRSS2 and ETS transcription factor genes in prostate cancer. Science 310: 644-648, 2005.

11. Urbinati G, Ali HM, Rousseau Q, Chapuis H, Desmaële D, Couvreur P and Massaad-Massade L: Antineoplastic effects of siRNA against TMPRSS2-ERG junction oncogene in prostate cancer. PLoS One 10: e0125277, 2015.

12. Urbinati G, de Waziers I, Slamiç M, Foussignière T, Ali HM, Desmaële D, Couvreur P and Massaad-Massade L: Knocking down TMPRSS2-ERG fusion oncogene by siRNA could be an alternative treatment to flutamide. Mol Ther Nucleic Acids 5: e301, 2016.

13. Shao L, Tekedereli I, Wang J, Yuca E, Tsang S, Sood A, Lopez-Berestein G, Ozpolat B and Ittmann M: Highly specific targeting of the TMPRSS2/ERG fusion gene using liposomal nanovectors. Clin Cancer Res 18: 6648-6657, 2012.

14. Wang X, Qiao Y, Asangani IA, Ateeq B, Poliakov A, Cieślik M, Pitchiaya S, Chakravarthi BVSK, Cao X, Jing X, et al: Development of peptidomimetic inhibitors of the ERG gene fusion product in prostate cancer. Cancer Cell 31: 532-548.e7, 2017.

15. Furusato B, Tan SH, Young D, Dobi A, Sun C, Mohamed AA, Thangapazham R, Chen Y, McMaster G, Sreenath T, et al: ERG oncoprotein expression in prostate cancer: Clonal progression of ERG-positive tumor cells and potential for ERG-based stratification. Prostate Cancer Prostatic Dis 13: 228-237, 2010.

16. Mehra R, Tomlins SA, Shen R, Nadeem O, Wang L, Wei JT, Pienta KJ, Ghosh D, Rubin MA, Chinnaiyan AM and Shah RB: Comprehensive assessment of TMPRSS2 and ETS family gene aberrations in clinically localized prostate cancer. Mod Pathol 20: 538-544, 2007.

17. Minner S, Gärtner M, Freudenthaler F, Bauer M, Kluth M, Salomon G, Heinzer H, Graefen M, Bokemeyer C, Simon R, et al: Marked heterogeneity of ERG expression in large primary prostate cancers. Mod Pathol 26: 106-116, 2013.

18. Tsourlakis MC, Stender A, Quaas A, Kluth M, Wittmer C, Haese A, Graefen M, Steurer S, Simon R, Korbel J, et al: Heterogeneity of ERG expression in prostate cancer: A large section mapping study of entire prostatectomy specimens from 125 patients. BMC Cancer 16: 641, 2016.

19. Minner S, Enodien M, Sirma H, Luebke AM, Krohn A, Mayer PS, Simon R, Tennstedt P, Müller J, Scholz L, et al: ERG status is unrelated to PSA recurrence in radically operated prostate cancer in the absence of antihormonal therapy. Clin Cancer Res 17: 5878-5888, 2011.

20. Fleischmann A, Saramäki OR, Zlobec I, Rotzer D, Genitsch V, Seiler R, Visakorpi T and Thalmann GN: Prevalence and prognostic significance of TMPRSS2-ERG gene fusion in lymph node positive prostate cancers. Prostate 74: 1647-1654, 2014.

21. Wise AM, Stamey TA, McNeal JE and Clayton JL: Morphologic and clinical significance of multifocal prostate cancers in radical prostatectomy specimens. Urology 60: 264-269, 2002.

22. Clement T, Swars H, Boerner N, Klose KJ, John H, Warnecke M and Weilemann LS: Venous occlusive disease of the liver-a rare pregnancy complication. Internist (Berl) 31: 297-300, 1990 (In German).

23. Barry M,Perner S, Demichelis F and Rubin MA: TMPRSS2-ERG fusion heterogeneity in multifocal prostate cancer: Clinical and biologic implications. Urology 70: 630-633, 2007.

24. Svensson MA, LaFargue CJ, MacDonald TY, Pflueger D, Kitabayashi N, Santa-Cruz AM, Garsha KE, Sathyanarayana UG, Riley JP, Yun CS, et al: Testing mutual exclusivity of ETS rearranged prostate cancer. Lab Invest 91: 404-412, 2011. 
25. Zhang S, Pavlovitz B, Tull J, Wang Y, Deng FM and Fuller C: Detection of TMPRSS2 gene deletions and translocations in carcinoma, intraepithelial neoplasia, and normal epithelium of the prostate by direct fluorescence in situ hybridization. Diagn Mol Pathol 19: 151-156, 2010.

26. Font-Tello A, Juanpere N, de Muga S, Lorenzo M, Lorente JA, Fumado L, Serrano L, Serrano S, Lloreta J and Hernández S: Association of ERG and TMPRSS2-ERG with grade, stage, and prognosis of prostate cancer is dependent on their expression levels. Prostate 75: 1216-1226, 2015.

27. Hagen RM, Adamo P, Karamat S, Oxley J, Aning JJ, Gillatt D, Persad R, Ladomery MR and Rhodes A: Quantitative analysis of ERG expression and its splice isoforms in formalin-fixed, paraffin-embedded prostate cancer samples: Association with seminal vesicle invasion and biochemical recurrence. Am J Clin Pathol 142: 533-540, 2014.

28. Hägglöf C, Hammarsten P, Strömvall K, Egevad L, Josefsson A, Stattin P, Granfors T and Bergh A: TMPRSS2-ERG expression predicts prostate cancer survival and associates with stromal biomarkers. PLoS One 9: e86824, 2014.

29. Klein EA, Falzarano SM, Maddala T, Cherbavaz D, Novotny WF, Millward C and Magi-Galluzzi C: Use of TMPRSS2-ERG gene rearrangement and quantitative ERG expression to predict clinical recurrence after radical prostatectomy. J Clin Oncol 29 (Suppl): S36, 2011.
30. Terry S, Nicolaiew N, Basset V, Semprez F, Soyeux P, Maillé P, Vacherot F, Ploussard G, Londoño-Vallejo A, de la Taille A and Allory Y: Clinical value of ERG, TFF3, and SPINK1 for molecular subtyping of prostate cancer. Cancer 121: 1422-1430, 2015.

31. Guo CC, Wang Y, Xiao L, Troncoso P and Czerniak BA: The relationship of TMPRSS2-ERG gene fusion between primary and metastatic prostate cancers. Hum Pathol 43: 644-649, 2012.

32. Perner S, Svensson MA, Hossain RR, Day JR, Groskopf J, Slaughter RC, Jarleborn AR, Hofer MD, Kuefer R, Demichelis F, et al: ERG rearrangement metastasis patterns in locally advanced prostate cancer. Urology 75: 762-767, 2010.

33. Tomlins SA, Laxman B, Dhanasekaran SM, Helgeson BE, Cao X, Morris DS, Menon A, Jing X, Cao Q, Han B, et al: Distinct classes of chromosomal rearrangements create oncogenic ETS gene fusions in prostate cancer. Nature 448: 595-599, 2007.

34. Carver BS, Tran J, Chen Z, Carracedo-Perez A, Alimonti A, Nardella C, Gopalan A, Scardino PT, Cordon-Cardo C, Gerald W and Pandolfi PP: ETS rearrangements and prostate cancer initiation. Nature 457: E1; discussion E2-E3, 2009.

c) (i) () $($ This work is licensed under a Creative Commons cc) $\mathrm{EY}$ NO NO Attribution-NonCommercial-NoDerivatives 4.0 International (CC BY-NC-ND 4.0) License. 